\title{
Pertuzumab in human epidermal growth-factor receptor 2-positive breast cancer: clinical and economic considerations
}

This article was published in the following Dove Press journal:

International Journal of Women's Health

16 May 2014

Number of times this article has been viewed

\section{Nathan WD Lamond Tallal Younis}

Department of Medicine, Dalhousie University at the Queen Elizabeth II Health Sciences Centre, Halifax, NS, Canada
Correspondence: Tallal Younis Department of Medicine, Dalhousie University at the Queen Elizabeth II Health Sciences Centre, Suite 454, Bethune Building, 1276 South Park Street, Halifax, NS B3H 2Y9, Canada

Tel +l 9024736054

Fax + I 9024736186

Email tallal.younis@cdha.nshealth.ca
Abstract: In the absence of specific therapy, the $15 \%-20 \%$ of breast cancers demonstrating human epidermal growth-factor receptor 2 (HER2) protein overexpression and/or gene amplification are characterized by a more aggressive phenotype and poorer prognosis compared to their HER2-negative counterparts. Trastuzumab (Herceptin), the first anti-HER2-targeted therapy, has been associated with improved survival outcomes in HER2-positive breast cancer. However, many patients with early stage disease continue to relapse, and metastatic disease remains incurable. In order to further improve these outcomes, several novel HER2-targeted agents have recently been developed. Pertuzumab (Perjeta), a monoclonal antibody against the HER2 dimerization domain, has also been associated with improved patient outcomes in clinical trials, and has recently been approved in combination with chemotherapy and trastuzumab for neoadjuvant therapy of early stage, HER2-positive breast cancer and first-line treatment of metastatic disease. This review briefly summarizes pertuzumab's clinical development as well as the published evidence supporting its use, and highlights some of the currently unanswered questions that will influence pertuzumab's incorporation into clinical practice.

Keywords: HER2/neu, clinical trials, drug development, novel therapies, targeted anticancer therapy

\section{Introduction}

Breast cancer is the most common nondermatologic malignancy in women worldwide and accounted for 1.38 million new diagnoses in 2011. ${ }^{1}$ Significant variation exists among breast cancers in both natural history and outcomes following therapy. Advances in our understanding of tumor biology have demonstrated a number of molecular and (recently) gene-expression profile characteristics that help explain this phenotypic heterogeneity. ${ }^{2}$ Human epidermal growth-factor receptor 2 (HER2; also known as HER2/neu and ERBB2) protein overexpression and/or gene amplification has been identified in $15 \%-20 \%$ of invasive breast cancers. ${ }^{3-6}$ In the absence of specific HER2-directed therapy, HER2-positive disease is characterized by a more aggressive phenotype and poorer prognosis compared to HER2-negative cancers. ${ }^{3-6}$

Trastuzumab (Herceptin ${ }^{\circledR}$; Genentech, San Francisco, CA, USA), the first US Food and Drug Administration (FDA)-approved agent to specifically target the HER2 protein, is a humanized monoclonal antibody against extracellular domain IV of the HER2 receptor (Figure 1). ${ }^{7}$ Trastuzumab has been associated with significant improvements in overall survival in both early and late-stage HER2-positive breast cancer settings. ${ }^{8-13}$ Despite this progress, however, many patients with early stage HER2-positive breast cancer continue to relapse, and metastatic HER2-positive disease remains incurable. 
Homodimerization

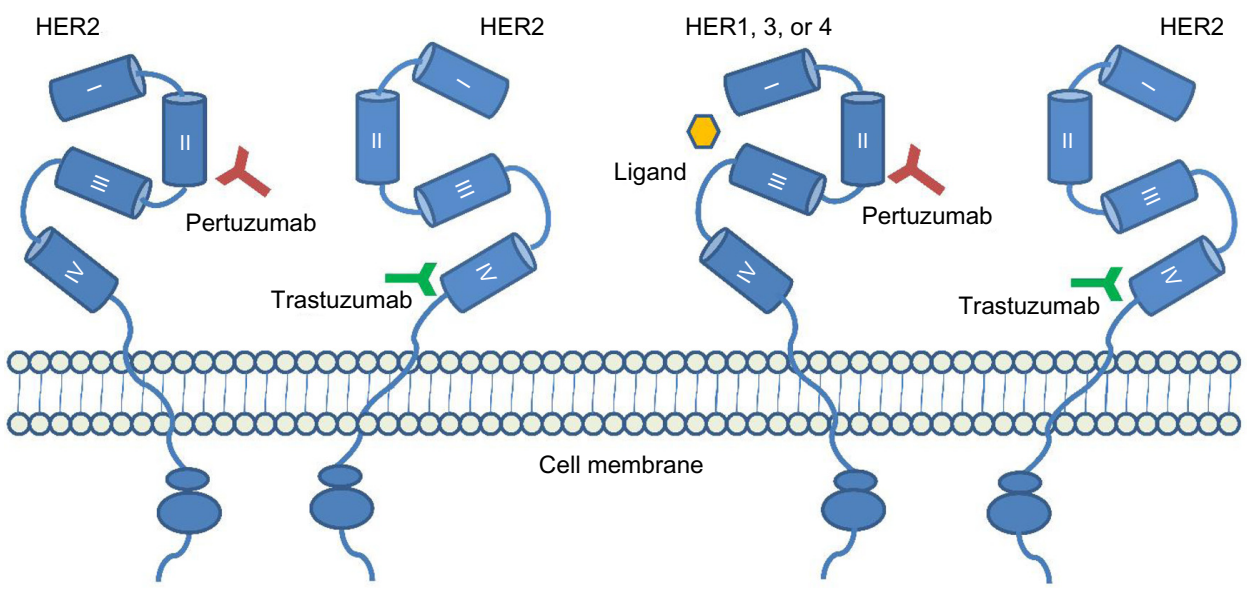

Heterodimerization

Intracellular tyrosine kinase domain

Abbreviation: HER, human epidermal growth-factor receptor.

Therefore, there is continued interest in the development of novel HER2-targeted agents to further improve patient outcomes in this breast cancer subgroup.

Pertuzumab (Perjeta ${ }^{\circledR}$; Genentech) is a humanized monoclonal antibody against extracellular domain II of the HER2 protein (Figure 1), and is the first in a new class of agents, HER dimerization inhibitors. ${ }^{14}$ The objective of this review is to summarize pertuzumab's clinical development as well as the published evidence supporting its use, and highlight some of the currently unanswered questions that will influence pertuzumab's incorporation into clinical practice. In preparation of this manuscript, we performed a Medline (PubMed) search for all English-language literature published prior to August 31, 2013 using the keywords "pertuzumab" and "breast cancer", which returned 193 publications. Further relevant reports were identified from the references of these articles.

\section{Mechanism of action and preclinical studies}

The HER family of proteins includes four transmembrane receptor-tyrosine kinases, namely HER1, HER2, HER3, and HER4. ${ }^{15-17}$ These proteins share a common mechanism in which intracellular tyrosine-kinase domains are activated through homo- or heterodimerization (receptor pairing), leading to initiation of several cell-signaling cascades. The process of dimerization is mediated by HER extracellular domain II, which in the majority of cases undergoes a conformational change allowing for dimerization upon ligand binding. HER2 is an exception, as it is constitutively able to dimerize in the absence of ligands, making it the preferred dimerization partner among HER proteins. ${ }^{18}$ Each individual HER dimerization pairing has unique cell-signaling properties with the HER2-HER3 heterodimer considered the most potent inducer of signal transduction, particularly for the activation of the phosphatidylinositide 3-kinase-AKTmammalian target of rapamycin pathway. ${ }^{18-20}$ Ultimately, HER-mediated cell signaling is involved in the regulation of a number of cellular processes, including proliferation, differentiation, migration, and apoptosis, ${ }^{16}$ which are central to cancer cell survival and therapeutic resistance. ${ }^{18,21}$

Whereas trastuzumab targets HER2 extracellular domain IV, pertuzumab is a fully humanized immunoglobulin $\mathrm{G}_{1}(\kappa)$ monoclonal antibody against HER2 extracellular domain II (Figure 1). ${ }^{14}$ By binding to an epitope within this domain, pertuzumab effectively blocks HER2 homo- and heterodimerization, ${ }^{14}$ thereby prohibiting HER tyrosinekinase activation and downstream cell signaling. ${ }^{22}$ Beyond interruption of HER-mediated cell signaling, pertuzumab has been shown to induce antibody-dependent cell-mediated cytotoxicity, similar to trastuzumab. ${ }^{14,21}$ These mechanisms, among others, result in significant antitumor activity demonstrated in both cell-line and xenograft models. ${ }^{23-26}$

Preclinical studies have consistently demonstrated a synergistic antitumor effect for a pertuzumab and trastuzumab combination. ${ }^{25,26}$ Interestingly, pertuzumab also appeared to inhibit tumor growth in a xenograft with low HER2 expression. ${ }^{23}$ While the former observation effectively foreshadows the clinical experience with pertuzumab to date, the latter has not yet translated into clinical benefit in 
the setting of HER2-negative disease ${ }^{27}$ Therefore, we have focused this review on the role of pertuzumab in HER2positive breast cancer, emphasizing the landmark clinical trials that have been completed or are in progress on this topic (Figure 2).

\section{Completed clinical trials Phase I trials}

The first Phase I trial was conducted among heavily pretreated patients with metastatic solid tumors to assess the safety and pharmacokinetics of pertuzumab monotherapy. ${ }^{28}$ This study included a dose-escalation schedule, with weight-based doses ranging from $0.5 \mathrm{mg} / \mathrm{kg}$ to $15 \mathrm{mg} / \mathrm{kg}$. A maximum dose was not identified, and pertuzumab was well tolerated overall. Pharmacokinetic data showed that the volume of distribution approximated the serum volume. With doses from $2 \mathrm{mg} / \mathrm{kg}$ to $15 \mathrm{mg} / \mathrm{kg}$, the elimination half-life ranged from 14 to 22 days, supporting a 3-week dosing schedule. Among 21 treated patients, two had partial responses, including patients with ovarian cancer and pancreatic islet-cell carcinoma, respectively. Stable disease lasting more than 10 weeks was observed in six additional patients.

Several other Phase I trials of pertuzumab monotherapy and combination regimens have been published in the advanced solid tumor setting. ${ }^{29-32}$ Taken together, these studies also suggested that pertuzumab was well tolerated, and a maximum dose was not reached. Pharmacokinetic data from these studies, as well as a subsequent analysis, confirmed that pertuzumab's half-life was approximately 3 weeks. ${ }^{29,33}$ These data also supported a fixed dosing schedule of $420 \mathrm{mg}$ administered intravenously (IV) every
3 weeks, preceded by a loading dose of $840 \mathrm{mg}$ in order to reach steady-state serum concentrations rapidly.

\section{Metastatic trials}

Initial Phase II trials in pretreated metastatic HER2-positive breast cancer patients investigated the addition of pertuzumab to trastuzumab, in an effort to overcome trastuzumab resistance (Table 1). ${ }^{34,35}$ The first of these was a small, single-arm trial in patients who had progressed on up to three prior lines of trastuzumab-based therapy for metastatic disease, including a median 82 weeks of prior trastuzumab therapy. ${ }^{34}$ The objectives of this study by Portera et al were to assess the safety and efficacy of dual HER2 blockade, with a focus on cardiac risk evaluation. The study's group sequential design included a planned sample size of 37 patients, with the option to stop accrual if two of the initial 12 enrolled subjects developed cardiac dysfunction. Accrual was stopped after only eleven patients, due to concern regarding cardiotoxicity. This will be discussed in more detail in the "Safety profile" section of this manuscript. Following the addition of pertuzumab in this small group, the objective response rate (ORR) was $18 \%$, and a further $27 \%$ of patients achieved $\geq 18$ weeks of disease stability (Table 1).

A second, larger Phase II study entitled B017929 also assessed the efficacy of combined trastuzumab and pertuzumab following progression on trastuzumab. ${ }^{35}$ In this multicenter, single-arm trial, dual HER2 blockade was continued until disease progression, treatment toxicity, or death. Enrolled patients had centrally confirmed HER2positive, metastatic breast cancer and had progressed on trastuzumab-based therapy as their most recent treatment for

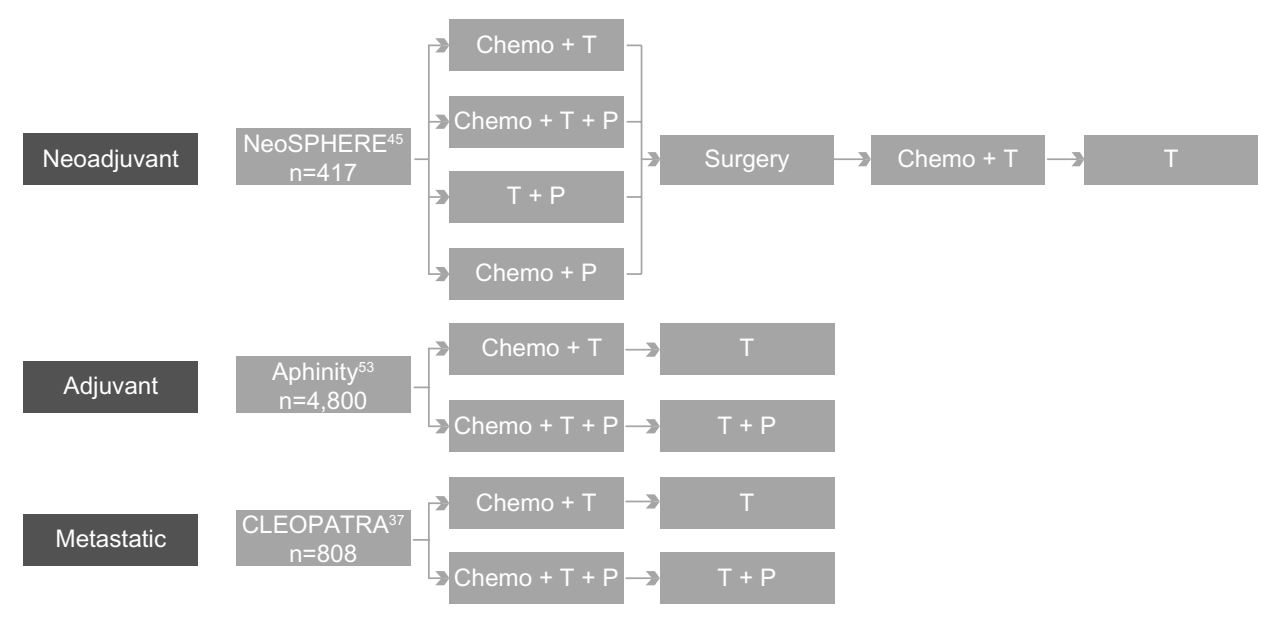

Figure 2 Landmark trials of pertuzumab in the adjuvant, neoadjuvant, and metastatic settings. The chemotherapy regimens utilized include docetaxel (CLEOPATRA and preoperatively in NEOSPHERE), fluorouracil/epirubicin/cyclophosphamide (postoperative in NEOSPHERE), and other center-specific approved regimens (Aphinity). Targeted therapies were delivered for a total of I year in the neoadjuvant and adjuvant trials or until progression in the metastatic trial. Abbreviations: Chemo, chemotherapy; T, trastuzumab; P, pertuzumab. 
Table I Clinical trials of pertuzumab in the metastatic setting

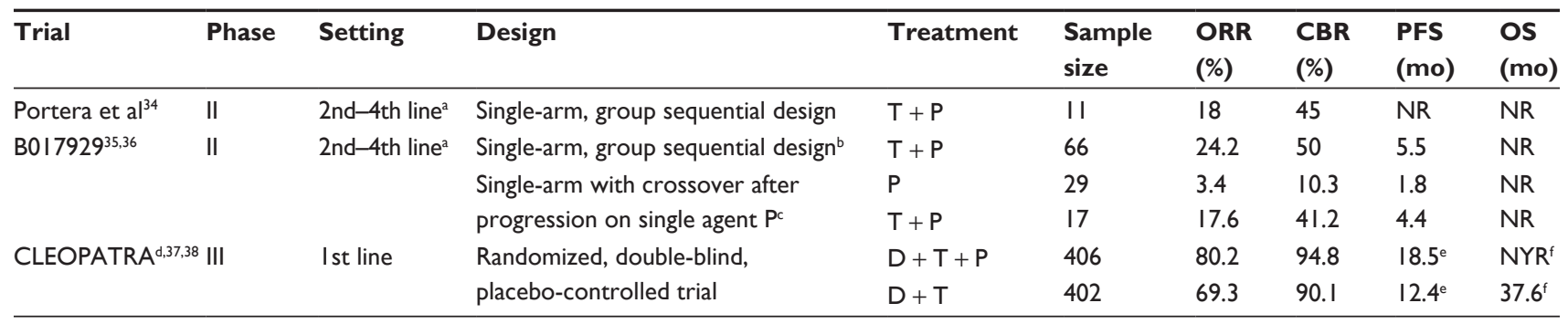

Notes: ${ }^{\mathrm{F}}$ Following progression on one or two lines of therapy, including T; ${ }^{\mathrm{b}}$ cohorts I and 2; ${ }^{\mathrm{c}}$ cohort 3; ${ }^{\mathrm{d}}$ the ORR and CBR results in CLEOPATRA are from primary analysis independent review data while PFS and OS are from 2 nd interim analysis - investigator-assessed data; ${ }^{\text {ehazard }}$ ratio $0.62, P<0.00 \mathrm{I}$; ' hazard ratio $0.66, P=0.0008$.

Abbreviations: ORR, overall response rate; CBR, clinical benefit rate; PFS, progression-free survival; mo, months; OS, overall survival; T, trastuzumab; P, pertuzumab; NR, not reported; D, docetaxel; NYR, not yet reached.

metastatic disease. Inclusion criteria allowed up to three prior chemotherapy regimens and required that study treatment be initiated 4-9 weeks after the subject's last dose of trastuzumab. The study's Simon-type, group sequential design included an interim analysis after enrollment of 24 evaluable patients (cohort 1), following which an additional group (cohort 2) was accrued based on demonstrated treatment activity. In all, 66 patients were enrolled with a median 16.2 months' prior trastuzumab treatment. Combination therapy using trastuzumab and pertuzumab was well tolerated, and again demonstrated activity in this setting. The observed ORR was 24\%, with five complete responses (CRs; 8\%). The clinical benefit rate, including the ORR and patients with stable disease for more than 6 months, was $50 \%$. Among responders, the median time to response was 2.6 months, no CR was seen before 6 months of therapy, and responses were seen in both visceral and nonvisceral disease. The median progression-free survival (PFS) and duration of response were 5.5 and 5.8 months, respectively.

Following the promising activity demonstrated in the first two cohorts of patients enrolled in B017929, the study protocol was amended, and a third cohort of patients was recruited (cohort 3 ). ${ }^{36}$ The purpose of this amendment was to investigate whether the efficacy of pertuzumab alone would be similar to that seen with dual HER2 blockade using trastuzumab and pertuzumab. Inclusion criteria for this group were identical to those of the original two cohorts, though study treatment differed (Table 1). In cohort 3 , patients who had progressed on trastuzumab were treated with pertuzumab monotherapy until disease progression or unacceptable toxicity. In patients tolerant of pertuzumab who developed progressive disease, trastuzumab was restarted in addition to pertuzumab at the investigator's discretion. There were 29 patients recruited with 87.2 weeks' mean prior trastuzumab treatment. Pertuzumab was well tolerated, but showed little activity as a monotherapy, with an ORR of $2.4 \%$ and median PFS of only 7.1 weeks (Table 1 ). Of the 29 patients who received pertuzumab, 17 subsequently had trastuzumab reintroduced with more substantial response rates, including an ORR of $17 \%$, a stable disease rate of $23.5 \%$, and a PFS of 17.4 weeks. Overall, these results suggest that the clinical benefit seen with the addition of pertuzumab to trastuzumab could be due to the synergistic impact of dual HER2 blockade, as opposed to pertuzumab anti-HER2 activity alone, seemingly confirming the observed preclinical synergy between these agents and pertuzumab's ability to "overcome trastuzumab resistance". ${ }^{25,26}$

The only Phase III trial of pertuzumab in the metastatic HER2-positive breast setting published to date examined the addition of pertuzumab to first-line treatment of docetaxel plus trastuzumab. ${ }^{37,38}$ The CLEOPATRA (CLinical Evaluation Of Pertuzumab And TRAstuzumab) registration trial, funded and sponsored by Hoffmann-La Roche (Basel, Switzerland), was an international, double-blind, randomized, placebo-controlled study (Table 1). Inclusion criteria allowed for prior endocrine therapy in the metastatic setting and prior chemotherapy with or without trastuzumab in the neo-/ adjuvant setting if the interval between treatment completion and diagnosis with metastatic disease was at least 12 months. Prior therapy was included as a stratification factor along with geographic region. Patients were randomized to receive docetaxel $75 \mathrm{mg} / \mathrm{m}^{2} \mathrm{IV}$, trastuzumab $8 \mathrm{mg} / \mathrm{kg}$ followed by $6 \mathrm{mg} / \mathrm{kg} \mathrm{IV}$, and standard-dose pertuzumab or matching placebo every 3 weeks for at least six recommended cycles. The number of docetaxel-chemotherapy cycles in the absence of disease progression was left to investigator discretion, while trastuzumab and pertuzumab (or placebo) were continued until disease progression or unmanageable toxicity. Crossover was not permitted at the time of progression. The primary end point was independently reviewed PFS in the intention-to-treat population. Secondary end points included overall survival (OS), ORR, and safety. Between February 2008 and July 
2010, 808 patients with centrally confirmed HER2-positive, unresectable locally recurrent, or metastatic breast cancer were enrolled. The baseline characteristics were well balanced among the two groups, and approximately $11 \%$ of patients had received trastuzumab previously.

In the primary analysis, conducted in May 2011 after a predefined number of events had been reached, ${ }^{37}$ the median PFS in the pertuzumab and placebo groups was 18.5 and 12.4 months, respectively (Table 2). This 6.1-month difference was clinically as well as statistically significant (hazard ratio [HR] $0.62,95 \%$ confidence interval $[\mathrm{CI}] 0.51-0.75 ; P<0.001)$, and was observed across all predefined subgroups. The ORR in the pertuzumab group was $80.2 \%$ compared to $69.3 \%$ in the placebo group (difference 10.8\%, 95\% CI 4.2-17.5; $P=0.001$ ). An interim analysis of OS conducted at this time showed fewer deaths in the pertuzumab group (HR 0.64, 95\% CI 0.47-0.88; $P=0.005$ ), but did not cross the predefined O'Brien-Fleming stopping boundary. Based on this, the trial continued and patients were not unblinded. The addition of pertuzumab was generally well tolerated (Table 3 ), and the median number of docetaxel cycles along with the median dose intensity were equal among groups.

A second interim analysis of OS from CLEOPATRA was performed in May 2012, 1 year after the primary analysis, as requested by European health authorities. ${ }^{38}$ At that time $267(69 \%)$ of the prespecified 385 events needed to trigger the final OS analysis had occurred. This analysis again showed fewer deaths in the pertuzumab group compared to the control arm (HR 0.66, 95\% CI 0.52-0.84; $P=0.0008$ ) and the HR for death crossed the predefined stopping boundary. Median OS had not been reached in the pertuzumab group, and was 37.6 months in the placebo group. Overall, approximately $70 \%$ of patients had received HER2-targeted agents following progression, including further trastuzumab (43\%), lapatinib (Tykerb ${ }^{\circledR}$; GlaxoSmithKline, Brentford, UK; 43\%), and trastuzumab emtansine (Kadcyla ${ }^{\circledR}$; Genentech; 10\%). Subsequent treatments were well balanced between groups, and no patients received pertuzumab off-study. Descriptive follow-up analysis showed that the PFS benefit had been maintained after an additional year of follow-up. Based on these results, Swain et al concluded that the addition of pertuzumab to first-line therapy with docetaxel and trastuzumab resulted in significant and clinically meaningful improvement in OS among women with HER2-positive metastatic breast cancer. ${ }^{38}$

A later publication of CLEOPATRA data reported its health-related quality-of-life (HRQoL) results. ${ }^{39}$ This evaluation utilized a well-validated questionnaire, the Functional Assessment of Cancer Therapy - Breast (FACT-B), which has established minimally important difference thresholds. ${ }^{40,41}$ The prespecified HRQoL end point of CLEOPATRA was the time from randomization until decline in HRQoL, as assessed by FACT-B, which was administered to subjects every 9 weeks. Results showed no difference among treatment groups, suggesting that the addition of pertuzumab to firstline treatment did not impair HRQoL. An exploratory, post hoc analysis using the breast cancer subscale of FACT-B suggested that pertuzumab-containing therapy may prolong time to clinically relevant worsening in breast cancer-specific symptoms.

The aforementioned positive results from CLEOPATRA have led to the recent approval of pertuzumab in addition to docetaxel plus trastuzumab for the first-line treatment of HER2-positive metastatic breast cancer by the European Medicines Agency, Health Canada, and the FDA. ${ }^{42-44}$

\section{Neoadjuvant trials}

To date, two Phase II trials examining pertuzumab therapy in the neoadjuvant setting have been published (Table 2). ${ }^{45,46}$ The NEOSPHERE (Neoadjuvant Study of Pertuzumab and

Table 2 Clinical trials of pertuzumab in the neoadjuvant setting

\begin{tabular}{|c|c|c|c|c|c|c|c|c|}
\hline \multirow[t]{2}{*}{ Trial } & \multirow[t]{2}{*}{ Phase } & \multirow[t]{2}{*}{ Arms } & \multirow[t]{2}{*}{$\mathbf{n}$} & \multirow[t]{2}{*}{ Preoperative treatment } & \multirow[t]{2}{*}{ \# cycles ${ }^{\mathrm{a}}$} & \multirow[t]{2}{*}{ Postoperative treatment ${ }^{b}$} & \multicolumn{2}{|c|}{ pCR (\%) } \\
\hline & & & & & & & Breast & Breast + Axilla \\
\hline \multirow[t]{4}{*}{ NeoSPHERE ${ }^{45}$} & II & A & 107 & $\mathrm{D}+\mathrm{T}$ & 4 & $(\mathrm{FEC}+\mathrm{T}) \times 3 \rightarrow \mathrm{T}$ & 29 & 21.5 \\
\hline & & B & 107 & $D+T+P$ & 4 & $(\mathrm{FEC}+\mathrm{T}) \times 3 \rightarrow \mathrm{T}$ & $45.8^{c}$ & 39.3 \\
\hline & & C & 107 & $\mathrm{~T}+\mathrm{P}$ & 4 & $(\mathrm{D}+\mathrm{T}) \times 4 \rightarrow(\mathrm{FEC}+\mathrm{T}) \times 3 \rightarrow \mathrm{T}$ & $16.8^{d}$ & 11.2 \\
\hline & & $\mathrm{D}$ & 96 & $D+P$ & 4 & $(\mathrm{FEC}+\mathrm{T}) \times 3 \rightarrow \mathrm{T}$ & $24^{e}$ & 17.7 \\
\hline \multirow[t]{3}{*}{ TRYPHAENA ${ }^{\mathrm{f}, 46}$} & & A & 73 & $(\mathrm{FEC}+\mathrm{T}+\mathrm{P}) \times 3 \rightarrow(\mathrm{D}+\mathrm{T}+\mathrm{P}) \times 3$ & 6 & $\mathrm{~T}$ & 61.6 & 50.7 \\
\hline & & B & 75 & $(\mathrm{FEC}) \times 3 \rightarrow(\mathrm{D}+\mathrm{T}+\mathrm{P}) \times 3$ & 6 & $\mathrm{~T}$ & 57.3 & 45.3 \\
\hline & & C & 77 & $\mathrm{D}+$ Carbo $+\mathrm{T}+\mathrm{P}$ & 6 & $\mathrm{~T}$ & 66.2 & 51.9 \\
\hline
\end{tabular}

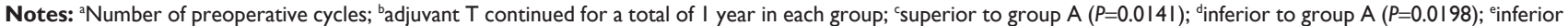
to group $B(P=0.003)$; ' no statistical comparison performed.

Abbreviations: $\mathrm{n}$, sample size; pCR, pathologic complete response; D, docetaxel; T, trastuzumab; FEC, fluorouracil/epirubicin/cyclophosphamide; P, pertuzumab; Carbo, carboplatin. 
Table 3 Common adverse events seen in CLEOPATRA

\begin{tabular}{|c|c|c|c|c|c|c|c|c|}
\hline & \multicolumn{2}{|c|}{$D+T+P(n=396)^{a}$} & \multicolumn{2}{|c|}{$D+T(n=408)^{a}$} & \multicolumn{2}{|c|}{$T+P(n=303)^{a}$} & \multicolumn{2}{|c|}{$T(n=260)^{a}$} \\
\hline & $\begin{array}{l}\text { Grade } \\
I-2\end{array}$ & $\begin{array}{l}\text { Grade } \\
3-4\end{array}$ & $\begin{array}{l}\text { Grade } \\
I-2\end{array}$ & $\begin{array}{l}\text { Grade } \\
3-4\end{array}$ & $\begin{array}{l}\text { Grade } \\
I-2\end{array}$ & $\begin{array}{l}\text { Grade } \\
3-4\end{array}$ & $\begin{array}{l}\text { Grade } \\
\mathrm{I}-2\end{array}$ & $\begin{array}{l}\text { Grade } \\
3-4\end{array}$ \\
\hline Diarrhea $^{b}$ & $24 \mid$ (59\%) & 37 (9\%) & |7| (43\%) & $20(5 \%)$ & $7 \mid(23 \%)$ & $7(2 \%)$ & 35 (13\%) & 0 \\
\hline Alopecia & $244(60 \%)$ & 0 & $236(60 \%)$ & $\mathrm{I}(<\mathrm{I} \%)$ & $4(1 \%)$ & 0 & $6(2 \%)$ & 0 \\
\hline Neutropenia & $16(4 \%)$ & $200(49 \%)$ & $15(4 \%)$ & $182(46 \%)$ & $8(3 \%)$ & 0 & $8(3 \%)$ & $4(2 \%)$ \\
\hline Nausea & I 74 (43\%) & $5(1 \%)$ & 166 (42\%) & $2(1 \%)$ & $29(10 \%)$ & $\mathrm{I}(<\mathrm{l} \%)$ & $29(11 \%)$ & 0 \\
\hline Fatigue & I 44 (35\%) & $9(2 \%)$ & 134 (34\%) & $13(3 \%)$ & $38(13 \%)$ & $2(1 \%)$ & $22(8 \%)$ & $3(1 \%)$ \\
\hline Rash $^{b}$ & $146(36 \%)$ & $3(1 \%)$ & $91(23 \%)$ & $3(1 \%)$ & $48(16 \%)$ & $\mathrm{I}(<\mathrm{I} \%)$ & $18(7 \%)$ & $\mathrm{I}(<\mathrm{l} \%)$ \\
\hline Anorexia & I 14 (28\%) & $7(2 \%)$ & $99(25 \%)$ & $6(2 \%)$ & $22(7 \%)$ & 0 & $9(3 \%)$ & $\mathrm{I}(<\mathrm{l} \%)$ \\
\hline Mucositis $^{b}$ & $106(26 \%)$ & $6(1 \%)$ & 75 (I9\%) & $4(1 \%)$ & $10(3 \%)$ & 0 & $4(2 \%)$ & 0 \\
\hline Asthenia & $100(25 \%)$ & $10(2 \%)$ & I I 4 (29\%) & $7(2 \%)$ & $36(12 \%)$ & $2(1 \%)$ & $21(8 \%)$ & $\mathrm{I}(<\mathrm{l} \%)$ \\
\hline Vomiting & $98(24 \%)$ & $6(1 \%)$ & 91 (23\%) & $6(2 \%)$ & $26(9 \%)$ & 0 & 17 (7\%) & 0 \\
\hline Peripheral edema & $97(24 \%)$ & $2(<1 \%)$ & I I 6 (29\%) & $3(1 \%)$ & $27(9 \%)$ & 0 & 31 (12\%) & 0 \\
\hline Pruritus $^{\mathrm{b}}$ & $66(16 \%)$ & 0 & $39(10 \%)$ & 0 & $34(11 \%)$ & 0 & I4 (5\%) & 0 \\
\hline Constipation & 63 (15\%) & 0 & 97 (24\%) & $4(1 \%)$ & 14 (5\%) & 0 & 17 (7\%) & $\mathrm{I}(<\mathrm{I} \%)$ \\
\hline Febrile neutropenia ${ }^{b}$ & 0 & $53(13 \%)$ & 0 & $29(7 \%)$ & 0 & 0 & 0 & 0 \\
\hline Dry skin ${ }^{\mathrm{b}}$ & 43 (I I\%) & 0 & $18(5 \%)$ & 0 & $8(3 \%)$ & 0 & $5(2 \%)$ & 0 \\
\hline
\end{tabular}

Notes: ${ }^{a}$ CLEOPATRA was a two-arm trial comparing $\mathrm{D}+\mathrm{T}+\mathrm{P}$ followed by $\mathrm{T}+\mathrm{P}$ and $\mathrm{D}+\mathrm{T}$ followed by $\mathrm{T}$; boccurring $>5 \%$ more frequently in the pertuzumab group. Reprinted from Lancet Oncol 20 13; 14(6). Swain SM, Kim SB, Cortés J, et al. Pertuzumab, trastuzumab, and docetaxel for HER2-positive metastatic breast cancer (CLEOPATRA study): overall survival results from a randomised, double-blind, placebo-controlled, phase 3 study, pages 46I-47I. Copyright (C) 20I3, with permission from Elsevier. ${ }^{38}$

Abbreviations: D, docetaxel; T, trastuzumab; P, pertuzumab; $n$, sample size.

Herceptin in an Early Regimen Evaluation) trial evaluated the addition of pertuzumab to neoadjuvant therapy consisting of docetaxel plus trastuzumab. This multicenter, open-label, proof-of-concept trial was funded by Hoffman-La Roche. Inclusion criteria included operable locally advanced or inflammatory centrally confirmed HER2-positive breast cancer with primary tumors larger than $2 \mathrm{~cm}$ in diameter. Patients were randomized into four arms and treated with a total of four preoperative treatment cycles administered every 3 weeks (Table 2). Those patients randomized to docetaxelcontaining arms received docetaxel $75 \mathrm{mg} / \mathrm{m}^{2} \mathrm{IV}$, escalating to $100 \mathrm{mg} / \mathrm{m}^{2}$ if tolerated. Postoperatively, patients received adjuvant chemotherapy with the FEC regimen (three cycles of fluorouracil $600 \mathrm{mg} / \mathrm{m}^{2}$, epirubicin $90 \mathrm{mg} / \mathrm{m}^{2}$, and cyclophosphamide $600 \mathrm{mg} / \mathrm{m}^{2}$ administered every 3 weeks IV) in arms A, B, and D, or with four cycles of docetaxel followed by FEC in group C. All patients received concurrent trastuzumab for 1 year. The primary end point was pathologic CR (pCR) in breast tissue alone (ypT0), though pCR in breast tissue and all sampled lymph-node (ypT0N0) rates were also reported. Patients were stratified by endocrine sensitivity (estrogen- and/ or progesterone-receptor positivity) and disease stage.

Between December 2007 and December 2009, 417 patients were enrolled internationally. Among this population, patients with endocrine-sensitive, locally advanced, and inflammatory disease represented $50 \%, 33 \%$, and $7 \%$, respectively. pCR rates differed among treatment arms (Table 2). Dual HER2 blockade plus docetaxel was associated with increased pCR rates compared to docetaxel plus trastuzumab (45.8\% versus $29.0 \%, P=0.0141)$ or docetaxel plus pertuzumab $(45.8 \%$ versus $24.0 \%, P=0.003)$. Conversely, dual HER2 blockade without chemotherapy was associated with inferior $\mathrm{pCR}$ rates when compared to docetaxel plus trastuzumab (16.8\% versus $29.0 \% ; P=0.0198)$. As expected, $\mathrm{pCR}$ rates were higher in patients with endocrine-insensitive disease compared with those with endocrine-sensitive cancers. The triple combination of pertuzumab plus docetaxel plus trastuzumab was associated with improved pCR rates compared to docetaxel plus trastuzumab alone in both endocrine-sensitive $(26.0 \%$ versus $20.0 \%)$ and endocrine-insensitive disease (63.2\% versus $36.8 \%$ ). The number of serious adverse events was similar among the arms that included chemotherapy and lower in the biologic therapy-alone group (arm C).

The TRYPHAENA (Trastuzumab Plus Pertuzumab in Neoadjuvant HER2-Positive Breast Cancer) trial also evaluated pertuzumab therapy in the neoadjuvant setting, with a focus on cardiac safety. ${ }^{46}$ This multicenter, randomized, open-label trial, funded by Hoffmann-La Roche, randomized patients into three treatment arms: dual HER2 blockade concurrent with anthracycline, sequential with anthracycline, and without anthracycline (Table 2). The treatments given in each arm were as follows: arm A, FEC-D (fluorouracil $500 \mathrm{mg} / \mathrm{m}^{2}$, epirubicin $100 \mathrm{mg} / \mathrm{m}^{2}$, cyclophosphamide $500 \mathrm{mg} / \mathrm{m}^{2}$ for three cycles followed by docetaxel $75 \mathrm{mg} / \mathrm{m}^{2}$ increased to $100 \mathrm{mg} / \mathrm{m}^{2}$ if tolerated for three cycles) chemotherapy with standard-dose trastuzumab plus pertuzumab given 
concurrently with all cycles; arm B, FEC-D chemotherapy with trastuzumab plus pertuzumab given concurrently with the last three cycles; or arm C, docetaxel $\left(75 \mathrm{mg} / \mathrm{m}^{2}\right)$, carboplatin (area under the plasma concentration-time curve of six), and trastuzumab plus pertuzumab given concurrently. All regimens were given IV every 3 weeks to a total of six preoperative cycles. Postoperative treatment included 1 year of adjuvant trastuzumab. This trial had similar inclusion criteria to NEOSPHERE. The primary end points in this case included 1) symptomatic left ventricular systolic dysfunction (LVSD) and 2) decreased left ventricular ejection fraction (LVEF) by $\geq 10 \%$ to $<50 \%$. The secondary end point was pCR in breast tissue alone (ypT0). A total of 225 patients were enrolled internationally between December 2009 and January 2011. Among this population, patients with endocrine-sensitive, locally advanced, and inflammatory disease represented approximately $50 \%, 20 \%-30 \%$, and $6 \%$, respectively. Median follow-up from time of enrolment was 20 months. The incidence of symptomatic LVSD and significant decreases in LVEF was low, and did not differ meaningfully across treatment arms (see further details in the "Safety profile" section of this manuscript). Arms A, B, and C were associated with high pCR rates of $61.6 \%, 57.3 \%$, and $66.2 \%$ in breast tissue alone (Table 2) and 50.7\%, 45.3\%, and $51.9 \%$, in breast and ipsilateral axillary nodes (ypT0N0), respectively. The authors concluded that all three neoadjuvant treatment regimens were safe and highly active.

Based on the aforementioned evidence, the FDA granted accelerated approval of pertuzumab use as part of neoadjuvant treatment for early breast cancer, contingent upon confirmatory results from an ongoing adjuvant trial. ${ }^{47}$

\section{Safety profile \\ Cardiotoxicity}

Due to the recognized potential for HER2-targeted agents to induce cardiotoxicity, cardiac safety has been the specific subject of several pertuzumab studies. ${ }^{34,46,48,49}$ The first reported Phase II trial of pertuzumab plus trastuzumab in patients with previously treated metastatic HER2-positive breast cancer was stopped due to potential cardiotoxicity. ${ }^{34}$ Inclusion criteria for this trial included a baseline LVEF $\geq 55 \%$ and no clinical evidence of congestive heart failure. Exclusion criteria included uncontrolled hypertension/arrhythmias, significant valvular lesions, prior myocardial infarction or angina pectoris requiring antianginal medication, poorly controlled diabetes mellitus, and prior treatment with $>360 \mathrm{mg} / \mathrm{m}^{2}$ of doxorubicin. Echocardiography was performed at baseline and repeated every 3 weeks. During the intended dual HER2 blockade in this trial, six of eleven patients experienced decreases in LVEF prompting trial discontinuation. Of these, two patients experienced decreases in LVEF $\geq 15 \%$ and one patient experienced symptomatic LVSD.

This concerning safety signal has not been replicated in subsequent trials with much larger sample sizes. Moreover, a pooled analysis of 598 patients from early phase pertuzumab trials suggested low rates of cardiotoxicity. ${ }^{49}$ In this study, the rates of asymptomatic LVEF decrease and symptomatic LVSD were approximately $5 \%$ and $1 \%$, respectively, and varied little depending upon whether pertuzumab was administered as a single agent or concurrent with nonanthracycline chemotherapy or trastuzumab. A reassuring cardiotoxicity profile was also observed in CLEOPATRA. ${ }^{37,38,48}$ In this trial, the incidence of cardiac adverse events was $16.4 \%$ in 402 patients treated with docetaxel plus trastuzumab plus placebo and $14.5 \%$ in 406 patients treated with docetaxel plus trastuzumab plus pertuzumab. The incidence of significant decline in LVEF, defined as a decline of $\geq 10 \%$ to a value of $<50 \%$, was $6.6 \%$ in the placebo group and $3.8 \%$ in the pertuzumab group. The rate of symptomatic LVSD among patients in the placebo and pertuzumab groups was $1.8 \%$ and $1.0 \%$, respectively.

The apparent cardiac safety of pertuzumab and trastuzumab with concurrent or sequential anthracycline-based chemotherapy has also been demonstrated in the neoadjuvant setting. ${ }^{46}$ In TRYPHAENA, significant decreases in LVEF (again defined as $\geq 10 \%$ to $<50 \%$ ) were infrequent, and only $0.4 \%$ of patients treated with concurrent pertuzumab plus trastuzumab plus anthracycline-based chemotherapy developed symptomatic LVSD during the neoadjuvant period. As a whole, the trial experience with single-agent and combination regimens including pertuzumab supports its cardiac safety in appropriately selected patients undergoing close monitoring. Routine assessments of LVEF continue to be recommended at baseline and every 3 months while on treatment.

\section{Other adverse effects}

Pertuzumab has demonstrated a favorable adverse-effect profile overall. During Phase I and II trials of pertuzumab with or without trastuzumab in the metastatic setting, the most common observed adverse events were diarrhea, asthenia, nausea, and rash. ${ }^{28-31,34-36}$ The majority of these events were mild, graded $1-2$ by the National Cancer Institute Common Toxicity Criteria. Pertuzumab also appears to be well tolerated when given concurrently with chemotherapy. ${ }^{37,45,46}$ In CLEOPATRA, pertuzumab was compared to placebo in 
addition to docetaxel plus trastuzumab. ${ }^{38}$ Adverse events present in at least $5 \%$ of this population are shown in Table 3 and divided based on occurrence during or after docetaxel therapy. Adverse events present at least 5\% more frequently in the pertuzumab group were diarrhea, rash, mucositis, febrile neutropenia, and dry skin. Among these, the only grade 3-4 events were febrile neutropenia, which occurred at rates of $13.8 \%$ and $7.7 \%$ in the pertuzumab and placebo groups, respectively. As expected, more adverse events took place during concurrent docetaxel treatment, including all episodes of febrile neutropenia.

Pertuzumab, like other monoclonal antibodies, has also been associated with hypersensitivity reactions that are generally mild and readily controlled with treatment, but may rarely be serious and life-threatening. No routine premedication is required. Finally, pertuzumab has been associated with fetal toxicity in animal studies, and is therefore not recommended for use in pregnancy or in women who may become pregnant. For this reason, a Phase IV observational study including pregnancy outcomes in women treated with pertuzumab is ongoing. ${ }^{50}$

\section{Ongoing trials}

Multiple ongoing trials are further examining the role of pertuzumab in HER2-positive breast cancer (Table 4). ${ }^{51-62}$ In the setting of first-line therapy for metastatic disease, several trials are currently open to accrual testing pertuzumab in combination with various other chemotherapy, endocrine, and targeted agents. A noteworthy Phase III trial has been designed based on promising results from a Phase IB/II trial of combined pertuzumab plus trastuzumab emtansine. ${ }^{32,57}$ MARIANNE (A Study of Trastuzumab Emtansine [TDM-1] Plus Pertuzumab/Pertuzumab Placebo Versus Trastuzumab [Herceptin] Plus a Taxane in Patients with Metastatic Breast Cancer) is a multicenter, randomized trial comparing three regimens in the firstline treatment of HER2-positive metastatic breast cancer: trastuzumab emtansine plus pertuzumab, trastuzumab emtansine plus placebo, and trastuzumab plus a taxane. ${ }^{57}$ Beyond first-line treatment, pertuzumab is being tested in addition to chemotherapy among patients who have not already received pertuzumab in PHEREXA (A Study of a Combination of Trastuzumab and Capecitabine with

Table 4 Ongoing pertuzumab clinical trials

\begin{tabular}{|c|c|c|c|c|c|c|c|}
\hline $\begin{array}{l}\text { Treatment } \\
\text { setting }\end{array}$ & Trial & Phase & Treatment groups & $\begin{array}{l}\text { Primary } \\
\text { end point }\end{array}$ & $\begin{array}{l}\text { Target } \\
\text { enrollment }\end{array}$ & $\begin{array}{l}\text { Estimated } \\
\text { completion }\end{array}$ & Comments \\
\hline \multirow[t]{2}{*}{ Neoadjuvant } & $\begin{array}{l}\text { Opti-HER Heart } \\
\text { (NCT01669239) }\end{array}$ & II & $\begin{array}{l}\text { Liposomal doxorubicin }+ \\
\text { paclitaxel }+T+P\end{array}$ & $\begin{array}{l}\text { Cardiac } \\
\text { safety }\end{array}$ & 83 & 2015 & \\
\hline & NCT0179619752 & ॥ & Paclitaxel $+\mathrm{T}+\mathrm{P}$ & $\mathrm{PCR}$ & 30 & 2015 & Inflammatory \\
\hline Adjuvant & $\begin{array}{l}\text { Aphinity } \\
\text { (NCT0I358877) }\end{array}$ & III & $\begin{array}{l}\text { I. Chemo }+\mathrm{T}+\mathrm{P} \\
\text { 2. Chemo }+\mathrm{T}\end{array}$ & DFS & 4,800 & 2023 & \\
\hline \multirow[t]{9}{*}{ Metastatic ${ }^{\mathrm{a}}$} & NCT0I $\left.27604\right|^{54}$ & II & Paclitaxel $+\mathrm{T}+\mathrm{P}$ & $\begin{array}{l}6 \text { month } \\
\text { PFS }\end{array}$ & 69 & 2014 & \\
\hline & $\begin{array}{l}\text { VELVET } \\
\text { (NCTOI565083) }\end{array}$ & II & Vinorelbine $+T+P$ & ORR & 210 & 2015 & \\
\hline & $\begin{array}{l}\text { PERTAIN }^{56} \\
\text { (NCTOI49I737) }\end{array}$ & ॥ & $\begin{array}{l}\text { I. } A l+T+P \\
\text { 2. } A l+T \\
\text { 3. Chemo }+T+P \rightarrow A l+T+P \\
\text { 4. Chemo }+T \rightarrow A I+T\end{array}$ & PFS & 250 & 2016 & \\
\hline & $\begin{array}{l}\text { MARIANNE }^{57} \\
(\text { NCTOIII20I84) }\end{array}$ & III & $\begin{array}{l}\text { I. T-DMI + P } \\
\text { 2. T-DMI } \\
\text { 3. Taxane }+\mathrm{T}\end{array}$ & $\begin{array}{l}\text { I. PFS } \\
\text { 2. AEs }\end{array}$ & $\mathrm{I}, 095$ & 2016 & \\
\hline & NCTOI $730833^{58}$ & ॥ & Nab-paclitaxel $+\mathrm{T}+\mathrm{P}$ & PFS & 45 & 2017 & \\
\hline & $\begin{array}{l}\text { PERUSE }^{59} \\
\text { (NCTOI572038) }\end{array}$ & Illb & Taxane $+\mathrm{T}+\mathrm{P}$ & Safety & $\mathrm{I}, 500$ & 2018 & \\
\hline & $\begin{array}{l}\text { HELENA }^{60} \\
\text { (NCTOI777958) }\end{array}$ & II & $\mathrm{T}+\mathrm{P}$ & PFS & 478 & 2020 & $\begin{array}{l}\text { Postadjuvant } \\
T\end{array}$ \\
\hline & NCTOI5974I1461 & ॥ & $\begin{array}{l}\text { I. Metronomic chemo }+\mathrm{T}+\mathrm{P} \\
\text { 2. } \mathrm{T}+\mathrm{P}\end{array}$ & PFS & 80 & NR & Elderly \\
\hline & $\begin{array}{l}\text { PHEREXA } \\
(\text { NCT0I26I42) }\end{array}$ & II & $\begin{array}{l}\text { I. Capecitabine }+\mathrm{T}+\mathrm{P} \\
\text { 2. Capecitabine }+\mathrm{T}\end{array}$ & PFS & 450 & 2017 & \\
\hline
\end{tabular}

Note: ${ }^{\text {aAll }}$ trials in first-line setting except PHEREXA, which is second-line.

Abbreviations: T, trastuzumab; P, pertuzumab; PCR, pathologic complete response; chemo, chemotherapy; DFS, disease-free survival; PFS, progression-free survival; ORR, overall response rate; $\mathrm{Al}$, aromatase inhibitor; T-DMI, trastuzumab emtansine; $\mathrm{AEs}$, adverse effects. 
or Without Pertuzumab in Patients with HER2-Positive Metastatic Breast Cancer). ${ }^{62}$

Pertuzumab is also currently undergoing evaluation in the adjuvant setting. Aphinity is a multicenter, randomized, double-blind, placebo-controlled trial of pertuzumab added to adjuvant treatment including chemotherapy plus trastuzumab..$^{53}$ The primary end point of this large, Phase III trial is invasive disease-free survival. With a reported target enrolment of 4,800 patients, the estimated completion date is 2023. In addition to assessing the value of pertuzumab in the adjuvant setting, clinically meaningful results from Aphinity will also help in interpretation of published surrogate outcomes from the neoadjuvant setting. ${ }^{45,46}$

\section{Clinical and economic perspectives}

Pertuzumab, the first in a new class of HER dimerization inhibitors, is clearly a promising agent for the treatment of HER2-positive breast cancer, and demonstrates substantial efficacy in combination with trastuzumab and chemotherapy. Dual HER2 blockade with this combination of targeted agents is well tolerated, allowing it to be safely combined with cytotoxic chemotherapies. However, important questions remain regarding pertuzumab's incorporation into routine clinical use.

\section{What is the optimal indication for pertuzumab?}

In the metastatic setting, the methodologically robust CLEOPATRA trial has shown significantly improved OS with pertuzumab when added to the first-line regimen of docetaxel plus trastuzumab. ${ }^{38}$ But is this regimen the ideal or only efficacious use for pertuzumab in metastatic HER2-positive breast cancer? Based on earlier phase data, it appears doubtful that pertuzumab's benefit is limited to the first-line setting or this particular regimen. ${ }^{34-36}$ Ongoing trials of pertuzumab in addition to regimens including various cytotoxic/targeted therapies in several lines of therapy will likely show significant benefits in other scenarios. ${ }^{55-57,62}$ Therefore, while its currently approved indication is first-line treatment in addition to docetaxel plus trastuzumab, time-limited use of pertuzumab in subsequent lines of therapy for those patients who did not receive pertuzumab in the first line may not be unreasonable.

The optimal combination and/or sequence with other novel HER2-targeted therapies, such as trastuzumab emtansine, is also yet to be determined. The three-arm MARIANNE trial of first-line treatment for metastatic HER2-positive breast cancer will examine trastuzumab emtansine with or without pertuzumab compared to taxane plus trastuzumab. ${ }^{57}$ However, as the study does not include a comparator arm of taxane plus trastuzumab plus pertuzumab, it is unlikely to provide a definite answer with regard to the preferred first-line regimen for women with HER2-positive metastatic breast cancer. Ultimately, in the not-too-distant future, clinicians may have a wealth of HER2-targeted therapies at their disposal that may be used in sequence or in combination based on prior therapies, potential adverse events, and drug access. Similar to the current paradigm for use of cytotoxic agents in metastatic breast cancer, current research is unlikely to identify a single ideal algorithm for use of HER2-targeted agents.

Results from NEOSPHERE and TRYPHAENA suggest that pertuzumab use in combination with neoadjuvant therapies is promising and safe, though questions persist. Does pertuzumab improve $\mathrm{pCR}$ rates when added to optimal neoadjuvant therapy? This question is difficult to answer, as a single optimal neoadjuvant therapy remains undefined. National Comprehensive Cancer Network guidelines recommend one of several chemotherapy regimens in addition to trastuzumab for neoadjuvant treatment. These preferred regimens include doxorubicin plus cyclophosphamide followed by taxane, taxane followed by FEC, or docetaxel plus carboplatin. The lowest number of cycles recommended among these regimens is six. Published experience with these regimens in combination with trastuzumab has reported pCR (ypT0N0) rates of up to $65.2 \% .{ }^{63}$ In NEOSPHERE, the backbone preoperative chemotherapy regimen consisted of single-agent docetaxel given for four cycles, which resulted in a pCR (ypT0N0) rate of $39.3 \%$ in combination with trastuzumab plus pertuzumab and only $21.5 \%$ in combination with trastuzumab alone. While cross-trial comparison is fraught with difficulties, these differences highlight the possibility that the control regimen in NEOSPHERE may not be an optimal neoadjuvant therapy comparator. This leads to the questions: can the observed benefit of pertuzumab in this trial be replaced by improving the regimen's cytotoxic backbone, or does pertuzumab add incremental benefit when combined with optimal neoadjuvant therapy? With these questions unanswered to date, further data supporting pertuzumab's role in the neoadjuvant setting are needed. Ultimately, results from Aphinity, the adjuvant Phase III trial designed to test the addition of pertuzumab to chemotherapy plus trastuzumab, may clarify pertuzumab's role in early stage disease, both adjuvant and neoadjuvant. Until further data are available, pertuzumab use as a component of neoadjuvant treatment has received temporary approval in some jurisdictions, and its use appears reasonable. 


\section{Does pertuzumab provide "good value for money"?}

Cost-effectiveness analyses (CEAs) and cost-utility analyses (CUAs) examine the economic cost of novel technologies in the context of their clinical benefit, and have become a standard part of new health-technology assessment in jurisdictions with publicly funded health care systems. CEAs and/or CUAs have the potential to improve the aggregate health of a population by allowing for the allocation of limited health care resources to technologies that provide the greatest value for money ${ }^{64}$ and could possibly mediate the rapidly rising costs of cancer care in the developed world. ${ }^{65}$

No academic CEAs or CUAs of pertuzumab exist in the literature to date. However as part of drug-approval assessments, pertuzumab's manufacturer has submitted its own economic evaluations of pertuzumab in addition to docetaxel plus trastuzumab for first-line treatment of metastatic HER2positive breast cancer. ${ }^{66,67}$ Limited details of one such evaluation have been publicly released following an application to the pan-Canadian Oncology Drug Review (pCODR) ${ }^{66}$ This submission included a CUA that employed economic modeling to estimate the monetary cost and quality-adjusted life years saved (QALYS) by pertuzumab in this setting. Model input included survival and HRQoL data from CLEOPATRA, as well as costs of drug acquisition, drug administration, adverse effects, and subsequent treatments. Using a submitted confidential price of pertuzumab, the pCODR's Economic Guidance Panel estimated that palliative pertuzumab therapy is associated with cost utility ranging from CA $\$ 262,263-\$ 303,726$ per QALYS, which is above the commonly reported North American threshold (CA $\$ 100,000$ per QALYS). ${ }^{68-70}$ Based on this estimate, the pCODR concluded that despite its demonstrated efficacy and safety, "pertuzumab could not be considered costeffective at the submitted confidential price". ${ }^{66}$

The UK's National Institute for Health and Care Excellence (NICE) has also reviewed a submission from the manufacturer for approval of pertuzumab in addition to docetaxel and trastuzumab as first-line treatment of metastatic HER2-positive breast cancer. ${ }^{71}$ In this submission, the listed price is noted to be $£ 2,395$ per $420 \mathrm{mg}$ vial. A similar CUA was performed, many details of which remain confidential. In the model, which met NICE methodological criteria, probabilistic sensitivity analysis suggested a $0 \%$ probability that pertuzumab is cost-effective at the commonly quoted UK threshold of $£ 30,000$ per QALYS. In sensitivity analysis, the model's cost-utility estimate was also noted to be highly sensitive to changes in pertuzumab cost. Based on this the committee's draft guidance states that "pertuzumab plus trastuzumab and docetaxel would not be a cost-effective use of NHS resources for treating HER2-positive metastatic or locally recurrent unresectable breast cancer compared with trastuzumab and docetaxel alone". ${ }^{71}$ The final NICE guidance is currently in development.

While regulatory bodies from two independent jurisdictions with publicly funded health care systems have concluded that pertuzumab does not appear to be cost-effective, it is important to recognize that pertuzumab's cost-effectiveness in these analyses was highly dependent on its drug acquisitioncosts. ${ }^{71}$ A more favorable cost-effectiveness for pertuzumab in this setting could be achieved by a lower drug price and/or identifying a subpopulation of patients who may derive larger benefit than those already reported in clinical trials. Based on the observation that the HER2-HER3 heterodimer is a potent cell-signaling inducer ${ }^{18-20}$ and hypotheses generating observations that response to pertuzumab-based therapy in ovarian cancer varies with HER3 expression, ${ }^{72}$ HER3 has been proposed as a rational candidate for predictive biomarker evaluations. ${ }^{73}$ To date, HER3 has yet to be identified as a discriminator of pertuzumab benefit in trials, and therefore the search for a predictive marker is ongoing. ${ }^{37,45}$

\section{Conclusion}

Pertuzumab, the first HER dimerization inhibitor, has shown significant promise for the treatment of early and late-stage HER2-positive breast cancer in combination with trastuzumab and/or chemotherapy. While many ongoing trials explore pertuzumab use in other settings, completed trials have demonstrated survival benefit when pertuzumab is added to docetaxel plus trastuzumab in first-line treatment of patients with metastatic disease, and improved $\mathrm{pCR}$ when pertuzumab is added to similar treatment in the neoadjuvant setting. However, the value for money associated with pertuzumab therapy at its current drug-acquisition cost appears unfavorable in jurisdictions with publicly funded health care systems. Therefore, access to this promising treatment may remain limited in certain jurisdictions based on economic factors, including its high drug-acquisition costs.

\section{Disclosure}

The authors report no conflicts of interest in this work.

\section{References}

1. Jemal A, Bray F, Center MM, Ferlay J, Ward E, Forman D. Global cancer statistics. CA Cancer J Clin. 2011;61(2):69-90.

2. Perou CM, Sørlie T, Eisen MB, et al. Molecular portraits of human breast tumours. Nature. 2000;406(6797):747-752.

3. Slamon DJ, Clark GM, Wong SG, Levin WJ, Ullrich A, McGuire WL. Human breast cancer: correlation of relapse and survival with amplification of the HER-2/neu oncogene. Science. 1987;235(4785):177-182. 
4. Slamon DJ, Godolphin W, Jones LA, et al. Studies of the HER-2/ neu proto-oncogene in human breast and ovarian cancer. Science. 1989;244(4905):707-712.

5. Wolff AC, Hammond ME, Schwartz JN, et al. American Society of Clinical Oncology/College of American Pathologists guideline recommendations for human epidermal growth factor receptor 2 testing in breast cancer. Arch Pathol Lab Med. 2007;131(1):18-43.

6. Ross JS, Slodkowska EA, Symmans WF, Pusztai L, Ravdin PM, Hortobagyi GN. The HER-2 receptor and breast cancer: ten years of targeted anti-HER-2 therapy and personalized medicine. Oncologist. 2009;14(4):320-368.

7. Hudis CA. Trastuzumab - mechanism of action and use in clinical practice. $N$ Engl J Med. 2007;357(1):39-51.

8. Slamon DJ, Leyland-Jones B, Shak S, et al. Use of chemotherapy plus a monoclonal antibody against HER2 for metastatic breast cancer that overexpresses HER2. N Engl J Med. 2001;344(11):783-792.

9. Marty M, Cognetti F, Maraninchi D, et al. Randomized phase II trial of the efficacy and safety of trastuzumab combined with docetaxel in patients with human epidermal growth factor receptor 2-positive metastatic breast cancer administered as first-line treatment: the M77001 study group. J Clin Oncol. 2005;23(19):4265-4274.

10. Piccart-Gebhart MJ, Procter M, Leyland-Jones B, et al. Trastuzumab after adjuvant chemotherapy in HER2-positive breast cancer. $N$ Engl J Med. 2005;353(16):1659-1672.

11. Joensuu H, Bono P, Kataja V, et al. Fluorouracil, epirubicin, and cyclophosphamide with either docetaxel or vinorelbine, with or without trastuzumab, as adjuvant treatments of breast cancer: final results of the FinHer Trial. J Clin Oncol. 2009;27(34):5685-5692.

12. Perez EA, Romond EH, Suman VJ, et al. Four-year follow-up of trastuzumab plus adjuvant chemotherapy for operable human epidermal growth factor receptor 2-positive breast cancer: joint analysis of data from NCCTG N9831 and NSABP B-31. J Clin Oncol. 2011;29(25): 3366-3373.

13. Slamon D, Eiermann W, Robert N, et al. Adjuvant trastuzumab in HER2positive breast cancer. $N$ Engl J Med. 2011;365(14):1273-1283.

14. Franklin MC, Carey KD, Vajdos FF, Leahy DJ, de Vos AM, Sliwkowski MX. Insights into ErbB signaling from the structure of the ErbB2-pertuzumab complex. Cancer Cell. 2004;5(4):317-328.

15. Yarden Y, Sliwkowski MX. Untangling the ErbB signalling network. Nat Rev Mol Cell Biol. 2001;2(2):127-137.

16. Yarden Y. The EGFR family and its ligands in human cancer. Signalling mechanisms and therapeutic opportunities. Eur J Cancer. 2001; 37 Suppl 4:S3-S8.

17. Schechter AL, Stern DF, Vaidyanathan L, et al. The neu oncogene: an erb-B-related gene encoding a 185,000-Mr tumour antigen. Nature. 1984;312(5994):513-516.

18. Tzahar E, Waterman $\mathrm{H}$, Chen $\mathrm{X}$, et al. A hierarchical network of interreceptor interactions determines signal transduction by $\mathrm{Neu}$ differentiation factor/neuregulin and epidermal growth factor. Mol Cell Biol. 1996;16(10):5276-5287.

19. Pinkas-Kramarski R, Soussan L, Waterman H, et al. Diversification of Neu differentiation factor and epidermal growth factor signaling by combinatorial receptor interactions. EMBO J. 1996;15(10):2452-2467.

20. Holbro T, Beerli RR, Maurer F, Koziczak M, Barbas CF 3rd, Hynes NE. The ErbB2/ErbB3 heterodimer functions as an oncogenic unit: ErbB2 requires ErbB3 to drive breast tumor cell proliferation. Proc Natl Acad Sci US A. 2003;100(15):8933-8938.

21. Adams CW, Allison DE, Flagella K, et al. Humanization of a recombinant monoclonal antibody to produce a therapeutic HER dimerization inhibitor, pertuzumab. Cancer Immunol Immunother. 2006;55(6): 717-727.

22. Baselga J. A new anti-ErbB2 strategy in the treatment of cancer: prevention of ligand-dependent ErbB2 receptor heterodimerization. Cancer Cell. 2002;2(2):93-95.

23. Agus DB, Akita RW, Fox WD, et al. Targeting ligand-activated ErbB2 signaling inhibits breast and prostate tumor growth. Cancer Cell. 2002;2(2):127-137.
24. Nahta R, Hung MC, Esteva FJ. The HER-2-targeting antibodies trastuzumab and pertuzumab synergistically inhibit the survival of breast cancer cells. Cancer Res. 2004;64(7):2343-2346.

25. Lee-Hoeflich ST, Crocker L, Yao E, et al. A central role for HER3 in HER2-amplified breast cancer: implications for targeted therapy. Cancer Res. 2008;68(14):5878-5887.

26. Scheuer W, Friess T, Burtscher H, Bossenmaier B, Endl J, Hasmann M. Strongly enhanced antitumor activity of trastuzumab and pertuzumab combination treatment on HER2-positive human xenograft tumor models. Cancer Res. 2009;69(24):9330-9336.

27. Gianni L, Lladó A, Bianchi G, et al. Open-label, phase II, multicenter, randomized study of the efficacy and safety of two dose levels of pertuzumab, a human epidermal growth factor receptor 2 dimerization inhibitor, in patients with human epidermal growth factor receptor 2-negative metastatic breast cancer. J Clin Oncol. 2010;28(7):1131-1137.

28. Agus DB, Gordon MS, Taylor C, et al. Phase I clinical study of pertuzumab, a novel HER dimerization inhibitor, in patients with advanced cancer. J Clin Oncol. 2005;23(11):2534-2543.

29. Attard G, Kitzen J, Blagden SP, et al. A phase Ib study of pertuzumab, a recombinant humanised antibody to HER2, and docetaxel in patients with advanced solid tumours. Br J Cancer. 2007;97(10):1338-1343.

30. Albanell J, Montagut C, Jones ET, et al. A phase I study of the safety and pharmacokinetics of the combination of pertuzumab (rhuMab 2C4) and capecitabine in patients with advanced solid tumors. Clin Cancer Res. 2008;14(9):2726-2731.

31. Yamamoto N, Yamada Y, Fujiwara Y, et al. Phase I and pharmacokinetic study of HER2-targeted rhuMAb 2C4 (pertuzumab, RO4368451) in Japanese patients with solid tumors. Jpn J Clin Oncol. 2009;39(4): 260-266.

32. Miller K, Gianni L, Andre F. A phase Ib/II trial of trastuzumab-DM1 (T-DM1) with pertuzumab (P) for women with HER2-positive, locally advanced or metastatic breast cancer (BC) who were previously treated with trastuzumab (T). J Clin Oncol. 2010;28(Suppl 15):1012.

33. Ng CM, Lum BL, Gimenez V, Kelsey S, Allison D. Rationale for fixed dosing of pertuzumab in cancer patients based on population pharmacokinetic analysis. Pharm Res. 2006;23(6):1275-1284.

34. Portera CC, Walshe JM, Rosing DR, et al. Cardiac toxicity and efficacy of trastuzumab combined with pertuzumab in patients with [corrected] human epidermal growth factor receptor 2-positive metastatic breast cancer. Clin Cancer Res. 2008;14(9):2710-2716.

35. Baselga J, Gelmon KA, Verma S, et al. Phase II trial of pertuzumab and trastuzumab in patients with human epidermal growth factor receptor 2-positive metastatic breast cancer that progressed during prior trastuzumab therapy. J Clin Oncol. 2010;28(7):1138-1144.

36. Cortés J, Fumoleau P, Bianchi GV, et al. Pertuzumab monotherapy after trastuzumab-based treatment and subsequent reintroduction of trastuzumab: activity and tolerability in patients with advanced human epidermal growth factor receptor 2-positive breast cancer. J Clin Oncol. 2012;30(14):1594-1600.

37. Baselga J, Cortés J, Kim SB, et al. Pertuzumab plus trastuzumab plus docetaxel for metastatic breast cancer. $N$ Engl J Med. 2012;366(2): 109-119.

38. Swain SM, Kim SB, Cortés J, et al. Pertuzumab, trastuzumab, and docetaxel for HER2-positive metastatic breast cancer (CLEOPATRA study): overall survival results from a randomised, double-blind, placebo-controlled, phase 3 study. Lancet Oncol. 2013;14(6):461-471.

39. Cortés J, Baselga J, Im YH, et al. Health-related quality-of-life assessment in CLEOPATRA, a phase III study combining pertuzumab with trastuzumab and docetaxel in metastatic breast cancer. Ann Oncol. 2013;24(10):2630-2635.

40. Brady MJ, Cella DF, Mo F, et al. Reliability and validity of the Functional Assessment of Cancer Therapy - Breast quality-of-life instrument. $J$ Clin Oncol. 1997;15(3):974-986.

41. Eton DT, Cella D, Yost KJ, et al. A combination of distribution- and anchor-based approaches determined minimally important differences (MIDs) for four endpoints in a breast cancer scale. J Clin Epidemiol. 2004;57(9):898-910. 
42. European Medicines Agency. Perjeta (pertuzumab). 2013. Available from: http://www.ema.europa.eu/ema/index.jsp?curl=pages/ medicines/human/medicines/002547/human_med_001628. jsp\&mid=WC0b01ac058001d124. Accessed October 7, 2013.

43. Health Canada. Summary basis of decision (SBD) for Perjeta. 2013. Available from: http://www.hc-sc.gc.ca/dhp-mps/prodpharma/sbdsmd/drug-med/sbd_smd_2013_perjeta_158419-eng.php. Accessed October 7, 2013.

44. Traynor K. FDA approves pertuzumab for breast cancer. Am J Health Syst Pharm. 2012;69(14):1178.

45. Gianni L, Pienkowski T, Im YH, et al. Efficacy and safety of neoadjuvant pertuzumab and trastuzumab in women with locally advanced, inflammatory, or early HER2-positive breast cancer (NeoSphere): a randomised multicentre, open-label, phase 2 trial. Lancet Oncol. 2012;13(1):25-32.

46. Schneeweiss A, Chia S, Hickish T, et al. Pertuzumab plus trastuzumab in combination with standard neoadjuvant anthracycline-containing and anthracycline-free chemotherapy regimens in patients with HER2positive early breast cancer: a randomized phase II cardiac safety study (TRYPHAENA). Ann Oncol. 2013;24(9):2278-2284.

47. US Food and Drug Administration. Approved drugs: pertuzumab injection. 2013. Available from: http://www.fda.gov/Drugs/ InformationOnDrugs/ApprovedDrugs/ucm370449.htm. Accessed January 16, 2014.

48. Swain SM, Ewer MS, Cortés J, et al. Cardiac tolerability of pertuzumab plus trastuzumab plus docetaxel in patients with HER2-positive metastatic breast cancer in CLEOPATRA: a randomized, doubleblind, placebo-controlled phase III study. Oncologist. 2013;18(3): 257-264.

49. Lenihan D, Suter T, Brammer M, Neate C, Ross G, Baselga J. Pooled analysis of cardiac safety in patients with cancer treated with pertuzumab. Ann Oncol. 2012;23(3):791-800.

50. Genentech. An Observational Study of Pregnancy and Pregnancy Outcomes in Women with Breast Cancer Treated with Herceptin, Perjeta in Combination with Herceptin, or Kadcyla During Pregnancy or Within 6 Months Prior to Conception (MotHER). Available from: http:// clinicaltrials.gov/show/NCT00833963. NLM identifier: NCT00833963. Accessed September 24, 2013.

51. SOLTI Breast Cancer Research Group. Study of Neoadjuvant Myocet ${ }^{\circledR}$, Paclitaxel, Pertuzumab, and Trastuzumab in HER2-positive Breast Cancer (Opti-HER Heart). Available from: http://clinicaltrials.gov/ show/NCT01669239. NLM identifier: NCT01669239. Accessed October 5, 2013.

52. Dana-Farber Cancer Institute. Paclitaxel + Trastuzumab + Pertuzumab as Pre-Op for BrCa. Available from: http://clinicaltrials.gov/show/ NCT01796197. NLM identifier: NCT01796197. Accessed October 5, 2013.

53. Hoffmann-La Roche. A Study of Pertuzumab in Addition to Chemotherapy and Herceptin (Trastuzumab) as Adjuvant Therapy in Patients with HER2-Positive Primary Breast Cancer. Available from: http://clinicaltrials.gov/show/NCT01358877. NLM identifier: NCT01358877. Accessed September 18, 2013.

54. Memorial Sloan-Kettering Cancer Center. Paclitaxel, Trastuzumab, and Pertuzumab in the Treatment of Metastatic HER2-Positive Breast Cancer. Available from: http://clinicaltrials.gov/show/ NCT01276041. NLM identifier: NCT01276041. Accessed October 5, 2013.

55. Hoffmann-La Roche. A Study of Pertuzumab in Combination with Herceptin (Trastuzumab) and Vinorelbine in First Line in Patients with Metastatic or Locally Advanced HER2-Positive Breast Cancer. Available from: http://clinicaltrials.gov/show/ NCT01565083. NLM identifier: NCT01565083. Accessed September 22, 2013.

56. Hoffmann-La Roche. A Study of Pertuzumab in Combination with Trastuzumab Plus an Aromatase Inhibitor in Patients with Hormone Receptor-Positive, Metastatic HER2-Positive Breast Cancer. Available from: http://clinicaltrials.gov/show/NCT01491737. NLM identifier: NCT01491737. Accessed September 22, 2013.
57. Hoffmann-La Roche. A Study of Trastuzumab Emtansine (T-DM1) Plus Pertuzumab/Pertuzumab Placebo Versus Trastuzumab [Herceptin] Plus a Taxane in Patients with Metastatic Breast Cancer (MARIANNE). Available from: http://clinicaltrials.gov/show/NCT01120184. NLM identifier: NCT01120184. Accessed September 18, 2013.

58. City of Hope Medical Center. Pertuzumab, Trastuzumab, and Paclitaxel Albumin-Stabilized Nanoparticle Formulation in Treating Patients with HER2-Positive Metastatic Breast Cancer. Available from: http:// clinicaltrials.gov/show/NCT01730833. NLM identifier: NCT01730833. Accessed October 5, 2013.

59. Hoffmann-La Roche. A Study of Pertuzumab in Combination with Herceptin (Trastuzumab) and a Taxane in First-Line Treatment in Patients with HER2-Positive Advanced Breast Cancer (PERUSE). Available from: http://clinicaltrials.gov/show/NCT01572038. NLM identifier: NCT01572038. Accessed October 5, 2013.

60. Hoffmann-La Roche. HELENA Study: An Observational Study of Perjeta (Pertuzumab) in First-Line Treatment in Patients with Her2Positive Advanced Breast Cancer after Adjuvant Herceptin Therapy. Available from: http://clinicaltrials.gov/show/NCT01777958. NLM identifier: NCT01777958. Accessed October 5, 2013.

61. European Organisation for Research and Treatment of Cancer. Elderly Metastatic Breast Cancer: Pertuzumab-Herceptin vs PertuzumabHerceptin-Metronomic Chemotherapy, Followed by T-DM1. Available from: http://clinicaltrials.gov/show/NCT01597414. NLM identifier: NCT01597414. Accessed October 5, 2013.

62. Hoffmann-La Roche. A Study of a Combination of Trastuzumab and Capecitabine with or Without Pertuzumab in Patients with HER2Positive Metastatic Breast Cancer (PHEREXA). Available from: http:// clinicaltrials.gov/show/NCT01026142. NLM identifier: NCT01026142. Accessed September 22, 2013.

63. Buzdar AU, Ibrahim NK, Francis D, et al. Significantly higher pathologic complete remission rate after neoadjuvant therapy with trastuzumab, paclitaxel, and epirubicin chemotherapy: results of a randomized trial in human epidermal growth factor receptor 2-positive operable breast cancer. J Clin Oncol. 2005;23(16):3676-3685.

64. Chambers JD, Lord J, Cohen JT, Neumann PJ, Buxton MJ. Illustrating potential efficiency gains from using cost-effectiveness evidence to reallocate Medicare expenditures. Value Health. 2013;16(4):629-638.

65. Smith TJ, Hillner BE. Bending the cost curve in cancer care. $N$ Engl $J$ Med. 2011;364(21):2060-2065.

66. Pan-Canadian Oncology Drug Review. Perjeta-Herceptin combo pack for MBC - details. 2013. Available from: http://www.pcodr.ca/wcpc/ portal/Home/FindaReview/PerjetaHerceptinMBC?afrLoop=230412 9379749000\&_afrWindowMode=0\&_adf.ctrl-state=fgpie73cf_134. Accessed September 20, 2013.

67. National Institute for Health and Care Excellence. Breast cancer (HER2 positive, metastatic) - pertuzumab (with trastuzumab and docetaxel) [ID523]. Available from: http://guidance.nice.org.uk/index. jsp?action=byId\&o=13815. Accessed September 20, 2013.

68. Hirth RA, Chernew ME, Miller E, Fendrick AM, Weissert WG. Willingness to pay for a quality-adjusted life year: in search of a standard. Med Decis Making. 2000;20(3):332-342.

69. Mason H, Baker R, Donaldson C. Willingness to pay for a QALY: past, present and future. Expert Rev Pharmacoecon Outcomes Res. 2008;8(6):575-582.

70. Murray CJ, Evans DB, Acharya A, Baltussen RM. Development of WHO guidelines on generalized cost-effectiveness analysis. Health Econ. 2000;9(3):235-251.

71. Breast cancer (HER2 positive, metastatic) - pertuzumab (with trastuzumab and docetaxel): appraisal consultation document. Natl Inst Health Care Excell. Available at: http://guidance.nice.org.uk/TAG/322/ Consultation/DraftGuidance. Accessed September 24, 2013.

72. Makhija S, Amler LC, Glenn D, et al. Clinical activity of gemcitabine plus pertuzumab in platinum-resistant ovarian cancer, fallopian tube cancer, or primary peritoneal cancer. J Clin Oncol. 2010;28(7):1215-1223.

73. Şendur MAN, Aksoy S, Zengin N. Pertuzumab plus trastuzumab in metastatic breast cancer. $N$ Engl J Med. 2012;366(14):1348; author reply $1349-1350$. 
International Journal of Women's Health

Dovepress

\section{Publish your work in this journal}

The International Journal of Women's Health is an international, peerreviewed open-access journal publishing original research, reports, editorials, reviews and commentaries on all aspects of women's healthcare including gynecology, obstetrics, and breast cancer. The manuscript management system is completely online and includes Visit http://www.dovepress.com/testimonials.php to read real quotes from published authors.

Submit your manuscript here: http://www.dovepress.com/international-journal-of-womens-health-journal 